\title{
Bridging kinetic plasma descriptions and single-fluid models
}

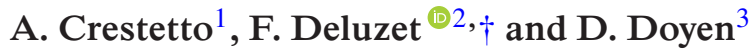 \\ ${ }^{1}$ Laboratoire de Mathématiques Jean Leray UMR 6629, 2 rue de la Houssinière, \\ F-44322 Nantes Cedex 3, France \\ ${ }^{2}$ UPS, INSA, UT1, UTM, Institut de Mathématiques de Toulouse, CNRS, Institut de Mathématiques de \\ Toulouse UMR 5219, Université de Toulouse, F-31062 Toulouse, France \\ ${ }^{3}$ Laboratoire d'Analyse et de Mathématiques Appliquées, CNRS, Laboratoire d'Analyse et de \\ Mathématiques Appliquées UMR 8050, Université de Marne-la-Vallée, 5 boulevard Descartes, Cité \\ Descartes - Champs-sur-Marne, F-77454 Marne-la-Vallée, France
}

(Received 15 April 2020; revised 16 July 2020; accepted 16 July 2020)

The purpose of this paper is to bridge kinetic plasma descriptions and low-frequency single-fluid models. More specifically, the asymptotics leading to magnetohydrodynamic regimes starting from the Vlasov-Maxwell system are investigated. The analogy with the derivation, from the Vlasov-Poisson system, of a fluid representation for ions coupled to the Boltzmann relation for electrons is also outlined. The aim is to identify asymptotic parameters explaining the transitions from one microscopic description to a macroscopic low-frequency model. These investigations provide groundwork for the derivation of multi-scale numerical methods, model coupling or physics-based preconditioning.

Key words: plasma simulation, fusion plasma, plasma flows

\section{Introduction}

The aim of this paper is to propose a continuation of the work initiated in Degond, Deluzet \& Navoret (2006), Degond et al. (2010) and Degond, Deluzet \& Doyen (2015) focusing on the derivation of asymptotic-preserving schemes for kinetic plasma descriptions in the quasi-neutral limit. The purpose of these numerical methods is to provide a quasi-neutral description of the plasma with no constraints on the simulation parameters related to the Debye length but with the ability to perform local up-scalings with non-neutral plasma descriptions. This brings a gain in the computational efficiency, since the discretization parameters can be set according to the physics of interest rather than the small scales (namely the Debye length) described by the model.

The methodology introduced in those former achievements is aimed to be generalized here to more singular limits. In this series of prior works, the limit models remain kinetic and the scales of interest are related to the electron dynamics. For instance, the quasi-neutral limit of the Vlasov-Maxwell system investigated in Degond et al. (2015) can be interpreted as a kinetic description of the electron magnetohydrodynamics (MHD) (Gordeev, Kingsep \& Rudakov 1994; Swanekamp et al. 1996; Cho \& Lazarian 2004),

$\dagger$ Email address for correspondence: fabrice.deluzet@math.univ-toulouse.fr 
accounting for the electron inertia, the massive ions being assumed at rest or slowly evolving. In the present paper, the objective is to go beyond the kinetic electron MHD with the aim of bridging the Vlasov-Maxwell system and MHD models. In MHD systems, the scales of interest are defined by the overall plasma dynamic which is governed by the ions, the fast scales associated with the electron inertia being filtered out from the equations.

The present work is therefore devoted to the derivation of a model hierarchy bridging either the Vlasov-Maxwell system and MHD models for magnetized plasmas, or the Vlasov-Poisson system and the electron adiabatic response, also referred to as the Boltzmann relation (see Langmuir (1929) and Tonks \& Langmuir $(1929 a, b)$ for seminal works and De Cecco et al. (2017) for numerical investigations), for electrostatic frameworks. A wide range of applications of the present investigations can be named, specifically low-variance particle-in-cell methods or more generally numerical discretization of kinetic models implementing a micro-macro decomposition of the distribution function. We refer for instance to Crestetto, Crouseilles \& Lemou (2012), Crouseilles \& Lemou (2011), Dimarco, Mieussens \& Rispoli (2014) and Lemou \& Mieussens (2008) for micro-macro methods, and to Degond, Dimarco \& Pareschi $(2011 b)$ for the moment guided method, fluid-preconditioned fully implicit methods (Chen, Chacón \& Barnes 2011; Chen et al. 2014; Chen \& Chacón 2015) and asymptotic-preserving numerical methods (Jin 1999; Degond \& Deluzet 2017). In these methods, macroscopic models are operated to either filter out the numerical noise of particle-in-cell methods (moment guided, micro-macro particle methods) or speed up the convergence of nonlinear implicit solvers (fully implicit particle-in-cell methods). The efficiency of these methods is closely related to that of the reduced models and adequacy of the kinetic plasma description. Another application can be envisioned with the hybrid coupling of particle-in-cell methods and MHD descriptions (Schumer et al. 2001; Daldorff et al. 2014) and more generally coupling strategies such as the current-coupling scheme and the pressure-coupling scheme (see Park et al. (1992), Tronci et al. (2014) and references therein).

The aim here is to clarify how the asymptotic parameters interact with each other and define reduced models, but also to relate these parameters to meaningful physical quantities. The MHD regime is sometimes derived by letting $\varepsilon_{0}$, the vacuum permittivity, go to zero (e.g. Jang \& Masmoudi 2012; Tronci \& Camporeale 2015) which is referred to as the full Maxwell or to the low-frequency pre-Maxwell asymptotic equations in Freidberg (2014, see $§ 2.3 .3)$. It is also common to let the electron to ion mass ratio go to zero to explain the vanishing of the electron inertia (Freidberg 2014; Klingenberg, Pirner \& Puppo 2016) in deriving either MHD modelling or the Boltzmann relation. Although the right asymptotic models are recovered by this means, these assumptions do not account for changes in the system characteristics that may explain a regime transition: the electron to ion mass ratio remains constant and the same property holds true for the vacuum permittivity.

The outline of the paper is the following. The plasma kinetic description is introduced in $\S 2$ together with the Maxwell system driving the evolution of the electromagnetic field. A dimensionless form of the system is stated in order to develop an asymptotic analysis and the derivation of reduced models. A hierarchy of quasi-neutral models is proposed in $\S 3$ for the Vlasov-Maxwell system. It encompasses fully kinetic, hybrid as well as single-fluid (MHD) plasma descriptions. The electrostatic framework is investigated in $\S 4$. The electrostatic limit of the Maxwell system is performed. A hierarchy of models, similar to that of the electromagnetic framework, is derived. Finally, a synthesis of these asymptotic analyses is proposed in $\S 5$ devoted to conclusions. 


\section{The Vlasov-Maxwell system in a dimensionless form}

\subsection{Objectives}

In this section, the purpose is to unravel a series of asymptotic limits bridging the gap between the Vlasov-Maxwell system and a MHD model. The difficulty is therefore to identify parameters explaining the transition from one description to the other and to relate these parameters to specific characteristics of the system. The tools mobilized to achieve this aim are based on the asymptotic analysis of the Vlasov-Maxwell system. Since the low-frequency plasma modelling is related to a fluid plasma description, the kinetic model is upgraded with collision operators. Therefore, the most refined modelling consists of a Vlasov equation for the electrons and the ions, augmented with a collision operator and coupled to the Maxwell system. Even if the physical model is non-collisional or weakly collisional, the transition towards a fluid limit is accounted for by a collisional process, thanks to a Bhatnager-Gross-Krook (BGK) operator. This choice of collision operator is questionable from a strict modelling viewpoint; nonetheless, the purpose here is to easily derive the fluid limit at a limited computational cost. In this respect the BGK collision operator is a good candidate. First, the whole collisional processes are considered, including both intra- and inter-species collisions. Nonetheless, only the minimal collisional process will be accounted for to derive a MHD regime from the kinetic model. This point will be outlined in the following sections. The introduction of non-dimensional quantities will naturally reveal dimensionless parameters in the equations. Letting some of these parameters go to zero shapes the hierarchy of models derived for the Vlasov-Maxwell system and bridging the gap with MHD models.

\subsection{The Vlasov-BGK-Maxwell system}

The most refined description of the plasma is constituted by two Vlasov equations, $f_{\mathrm{i}}$ and $f_{\mathrm{e}}$ being the ion and electron distribution functions:

$$
\begin{gathered}
\partial_{t} f_{\mathrm{i}}+v \cdot \nabla_{x} f_{\mathrm{i}}+\frac{q}{m_{\mathrm{i}}}(E+v \times B) \cdot \nabla_{v} f_{\mathrm{i}}=\mathcal{Q}_{\mathrm{i}}, \\
\partial_{t} f_{\mathrm{e}}+v \cdot \nabla_{x} f_{\mathrm{e}}-\frac{q}{m_{\mathrm{e}}}(E+v \times B) \cdot \nabla_{v} f_{\mathrm{e}}=\mathcal{Q}_{\mathrm{e}}
\end{gathered}
$$

In these equations, $q$ is the elementary charge and $m_{\alpha}$ is the mass of the species $\alpha$ ( $\alpha=\mathrm{e}$ for the electrons and $\mathrm{i}$ for the ions). The BGK collision operator $\mathcal{Q}_{\alpha}$ is given by (Huba 2011)

$$
\left.\begin{array}{c}
\mathcal{Q}_{\alpha}=\mathcal{Q}_{\alpha \alpha}+\mathcal{Q}_{\alpha \beta}, \\
\mathcal{Q}_{\alpha \alpha}=v_{\alpha \alpha}\left(\mathcal{M}_{n_{\alpha}, u_{\alpha}, T_{\alpha}}-f_{\alpha}\right), \quad \mathcal{Q}_{\alpha \beta}=v_{\alpha \beta}\left(\overline{\mathcal{M}}_{n_{\alpha}, \bar{u}_{\beta}, \bar{T}_{\beta}}-f_{\alpha}\right) .
\end{array}\right\}
$$

Here $v_{\alpha \alpha}$ and $v_{\alpha \beta}$ are the like-particle and inter-species collision frequencies which can be defined as (Degond 2007; Spatschek 2012)

$$
\begin{array}{ll}
v_{\mathrm{ii}}=K_{0} \frac{n_{\mathrm{i}}}{\left(k_{\mathrm{B}} T_{\mathrm{i}}\right)^{3 / 2}} \frac{\sqrt{2}}{\sqrt{m_{\mathrm{i}}}}, \quad v_{\mathrm{ie}}=K_{0} \frac{n_{\mathrm{e}}}{\left(k_{\mathrm{B}} T_{\mathrm{i}}\right)^{3 / 2}} \frac{\sqrt{m_{\mathrm{e}}}}{m_{\mathrm{i}}}, \\
v_{\mathrm{ee}}=K_{0} \frac{n_{\mathrm{e}}}{\left(k_{\mathrm{B}} T_{\mathrm{e}}\right)^{3 / 2}} \frac{\sqrt{2}}{\sqrt{m_{\mathrm{e}}}}, \quad v_{\mathrm{ei}}=K_{0} \frac{n_{\mathrm{i}}}{\left(k_{\mathrm{B}} T_{\mathrm{e}}\right)^{3 / 2}} \frac{1}{\sqrt{m_{\mathrm{e}}}},
\end{array}
$$


where

$$
K_{0}=C\left(\frac{q^{2}}{4 \pi \epsilon_{0}}\right)^{2} \ln (\Lambda),
$$

$C$ denoting a constant with a magnitude equal to one, $\ln (\Lambda)$ the Coulomb logarithm and the ions being assumed mono-charged.

The Maxwellians $\mathcal{M}_{n_{\alpha}, u_{\alpha}, T_{\alpha}}$ and $\overline{\mathcal{M}}_{n_{\alpha}, \bar{u}_{\beta}, \bar{T}_{\beta}}$ are defined as

$$
\begin{aligned}
& \mathcal{M}_{n_{\alpha}, u_{\alpha}, T_{\alpha}}=n_{\alpha}(x, t)\left(\frac{m_{\alpha}}{2 \pi k_{\mathrm{B}} T_{\alpha}(x, t)}\right)^{D_{v} / 2} \exp \left(-\frac{m_{\alpha}\left|u_{\alpha}(x, t)-v\right|^{2}}{2 k_{\mathrm{B}} T_{\alpha}(x, t)}\right), \\
& \overline{\mathcal{M}}_{n_{\alpha}, \bar{u}_{\beta}, \bar{T}_{\beta}}=n_{\alpha}(x, t)\left(\frac{m_{\alpha}}{2 \pi k_{\mathrm{B}} \bar{T}_{\beta}(x, t)}\right)^{D_{v} / 2} \exp \left(-\frac{m_{\alpha}\left|\bar{u}_{\beta}(x, t)-v\right|^{2}}{2 k_{\mathrm{B}} \bar{T}_{\beta}(x, t)}\right),
\end{aligned}
$$

$D_{v}$ denoting the dimension of the velocity space and $k_{\mathrm{B}}$ is the Boltzmann constant. The Maxwellian parameters $n_{\alpha}, u_{\alpha}$ and $T_{\alpha}$ are the density, mean velocity and temperature associated with the distribution function $f_{\alpha}$ and defined as

$$
n_{\alpha}=\int_{\Omega_{v}} f_{\alpha} \mathrm{d} v, \quad n_{\alpha} u_{\alpha}=\int_{\Omega_{v}} v f_{\alpha} \mathrm{d} v, \quad \frac{1}{\gamma-1} n_{\alpha} k_{\mathrm{B}} T_{\alpha}=\frac{m_{\alpha}}{2} \int_{\Omega_{v}}|v-u|^{2} f_{\alpha} \mathrm{d} v,
$$

with $\gamma$ the specific heat ratio whose value depends on the dimensionality of the velocity space $D_{v}$ through

$$
\gamma-1=\frac{2}{D_{v}}
$$

The collision operators verify the following conservation properties:

$$
\begin{gathered}
\int \mathcal{Q}_{\alpha \alpha} m_{\alpha}\left(\begin{array}{c}
1 \\
v \\
|v|^{2}
\end{array}\right) \mathrm{d} v=0 \\
\int \mathcal{Q}_{\alpha \beta} m_{\alpha}\left(\begin{array}{c}
1 \\
v \\
|v|^{2}
\end{array}\right) \mathrm{d} v+\int \mathcal{Q}_{\beta \alpha} m_{\beta}\left(\begin{array}{c}
1 \\
v \\
|v|^{2}
\end{array}\right) \mathrm{d} v=0 .
\end{gathered}
$$

The temperature and the mean velocity $\left(\bar{u}_{\beta}, \bar{T}_{\beta}\right)$ in the inter-species collision operator expression $(2.5 b)$ should be chosen with care in order to guarantee the total momentum and energy conservation. Indeed the identities

$$
\begin{gathered}
\int \mathcal{Q}_{\alpha \beta} m_{\alpha} v \mathrm{~d} v=v_{\alpha \beta} m_{\alpha} n_{\alpha}\left(\bar{u}_{\beta}-u_{\alpha}\right), \\
\int \mathcal{Q}_{\alpha \beta} m_{\alpha} \frac{|v|^{2}}{2} \mathrm{~d} v=v_{\alpha \beta}\left(\frac{D_{v}}{2} n_{\alpha} k_{\mathrm{B}}\left(\bar{T}_{\beta}-T_{\alpha}\right)+\frac{1}{2} m_{\alpha} n_{\alpha}\left(\left|\bar{u}_{\beta}\right|^{2}-\left|u_{\alpha}\right|^{2}\right)\right)
\end{gathered}
$$

hold true for the operators defined by (2.5). The trivial choice $\left(\bar{u}_{\beta}, \bar{T}_{\beta}\right)=\left(u_{\beta}, T_{\beta}\right)$ does ensure the plasma total momentum conservation, provided that $v_{\mathrm{ei}} m_{\mathrm{e}} n_{\mathrm{e}}=v_{\mathrm{ie}} m_{\mathrm{i}} n_{\mathrm{i}}$. However, in this case, the plasma total energy is not conserved. We refer to Greene (1973) for a seminal work, as well as to Klingenberg et al. (2016) and references therein for recent 
advances, on the choice of these parameters compliant with the desired properties $(2.8 b)$ of the inter-species collision operators, therefore providing the conservation of the plasma momentum and energy.

The electromagnetic field $(E, B)$ evolution is driven by the Maxwell system:

$$
\begin{gathered}
\frac{1}{c^{2}} \partial_{t} E-\nabla_{x} \times B=-\mu_{0} J, \\
\partial_{t} B+\nabla_{x} \times E=0, \\
\nabla_{x} \cdot E=\frac{\rho}{\epsilon_{0}}, \\
\nabla_{x} \cdot B=0,
\end{gathered}
$$

where $c$ is the speed of light, $\mu_{0}$ the vacuum permeability and $\epsilon_{0}$ the vacuum permittivity verifying $\mu_{0} \epsilon_{0} c^{2}=1$. The Maxwell sources are the particle currents and densities

$$
\begin{gathered}
\rho=q\left(n_{\mathrm{i}}-n_{\mathrm{e}}\right), \\
J=q\left(n_{\mathrm{i}} u_{\mathrm{i}}-n_{\mathrm{e}} u_{\mathrm{e}}\right) .
\end{gathered}
$$

The definition of the collision frequencies as stated by (2.4) relates to different time scales. Indeed, because of their different masses, ions and electrons are not equally affected by collisions. These properties are more clearly emphasized working with dimensionless variables as proposed in the next section.

\subsection{Scaling of the Vlasov-Maxwell system}

The equations are written with dimensionless quantities in order to easily identify different regimes. The scaling is introduced under a priori assumptions that the electronic and ionic temperatures, densities and mean velocities are comparable with a magnitude denoted $T_{0}$, $n_{0}$ and $u_{0}$. These scales define the typical Debye length as well as the electron plasma period:

$$
\lambda_{\mathrm{D}}=\sqrt{\frac{\epsilon_{0} k_{\mathrm{B}} T_{0}}{q^{2} n_{0}}}, \quad \tau_{\mathrm{pe}}=\sqrt{\frac{m_{\mathrm{e}} \epsilon_{0}}{q^{2} n_{0}}} .
$$

We denote by $x_{0}$ and $t_{0}$ the characteristic space and time scales of the phenomena observed, which yields the velocity of interest $\vartheta_{0}=x_{0} / t_{0}$. The magnitude of the thermal velocity for the species $\alpha$ is denoted $v_{0, \alpha}$ with $v_{0, \alpha}=k_{\mathrm{B}} T_{0} / m_{\alpha}$. Due to the different masses, the thermal velocity of the electrons is not that of the ions. The reference thermal velocity $v_{0}$ will be defined by the ion one $v_{0}^{2}=k_{\mathrm{B}} T_{0} / m_{\mathrm{i}}$, hence $v_{0, \mathrm{e}}=v_{0} / \varepsilon$ and $v_{0, \mathrm{i}}=v_{0}$, where $\varepsilon^{2}=m_{\mathrm{e}} / m_{\mathrm{i}}$. Finally, the scale of the electromagnetic field is denoted $\left(E_{0}, B_{0}\right)$, the particle current scale being defined as $J_{0}=q n_{0} u_{0}$. The dimensionless variables are defined according to

$$
\begin{gathered}
x^{*}=\frac{x}{x_{0}}, \quad t^{*}=\frac{t}{t_{0}}, \quad v^{*}=\frac{v}{v_{0, \alpha}}, \quad f^{*}=\frac{f}{n_{0} /\left(v_{0, \alpha}\right)^{D_{v}}}, \quad n^{*}=\frac{n}{n_{0}}, \quad J^{*}=\frac{J}{q n_{0} u_{0}}, \\
E^{*}=\frac{E}{E_{0}}, \quad B^{*}=\frac{B}{B_{0}},
\end{gathered}
$$

the collision frequencies verifying

$$
v_{\mathrm{ee}, 0}=v_{\mathrm{ei}, 0}=\frac{1}{\varepsilon} v_{\mathrm{ii}, 0}, \quad \nu_{\mathrm{ie}, 0}=\varepsilon v_{\mathrm{ii}, 0}, \quad \varepsilon=\sqrt{\frac{m_{\mathrm{e}}}{m_{\mathrm{i}}}} .
$$


On the fastest time scales, the electron distribution function relaxes towards a Maxwellian. On the same time scale, the electron mean velocity and temperature relax towards those of the ions. The relaxation of the ionic distribution function towards the local equilibrium is slower, by a factor $\varepsilon^{-1}=\sqrt{m_{\mathrm{i}} / m_{\mathrm{e}}}$. Finally, the ions are almost unaffected by the collisions with the electrons. The relaxation of the ionic distribution function towards that of the electrons defines the largest time scale, by a factor $\varepsilon^{-1}$ compared to the relaxation towards the thermodynamical equilibrium.

The dimensionless ionic and electronic Vlasov equations can be rewritten as (keeping the same notations for dimensionless variables)

$$
\begin{aligned}
& \xi \partial_{t} f_{\mathrm{i}}+v \cdot \nabla_{x} f_{\mathrm{i}}+\eta\left(E+\frac{\beta}{\xi} v \times B\right) \cdot \nabla_{v} f_{\mathrm{i}}=\frac{\xi}{\kappa}\left(v_{\mathrm{ii}}\left(\mathcal{M}_{n_{\mathrm{i}}, u_{\mathrm{i}}, T_{\mathrm{i}}}-f_{\mathrm{i}}\right)+\varepsilon v_{\mathrm{ie}}\left(\overline{\mathcal{M}}_{n_{\mathrm{i}}, u_{\mathrm{e}}, T_{\mathrm{e}}}-f_{\mathrm{i}}\right)\right), \\
& \xi \varepsilon \partial_{t} f_{\mathrm{e}}+v \cdot \nabla_{x} f_{\mathrm{e}}-\eta\left(E+\frac{\beta}{\varepsilon \xi} v \times B\right) \cdot \nabla_{v} f_{\mathrm{e}}=\frac{\xi}{\kappa}\left(v_{\mathrm{ee}}\left(\mathcal{M}_{n_{\mathrm{e}}, u_{\mathrm{e}}, T_{\mathrm{e}}}-f_{\mathrm{e}}\right)+v_{\mathrm{ei}}\left(\overline{\mathcal{M}}_{n_{\mathrm{e}}, u_{\mathrm{i}}, T_{\mathrm{i}}}-f_{\mathrm{e}}\right)\right),
\end{aligned}
$$

together with the dimensionless Maxwell system written

$$
\begin{gathered}
\lambda^{2} \eta\left(\alpha^{2} \frac{\partial E}{\partial t}-\beta \nabla_{x} \times B\right)=-\alpha^{2} \frac{M}{\xi} J, \\
\beta \partial_{t} B+\nabla_{x} \times E=0, \\
\lambda^{2} \eta \nabla_{x} \cdot E=n_{\mathrm{i}}-n_{\mathrm{e}}, \\
\nabla_{x} \cdot B=0, \\
J=n_{\mathrm{i}} u_{\mathrm{i}}-n_{\mathrm{e}} u_{\mathrm{e}} .
\end{gathered}
$$

This system is written using the following dimensionless parameters:

$\varepsilon^{2}=\frac{m_{\mathrm{e}}}{m_{\mathrm{i}}}$ the ratio of the electronic and ionic masses,

$\lambda=\frac{\lambda_{\mathrm{D}}}{x_{0}}$ the scaled Debye length,

$M=\frac{u_{0}}{v_{0}}$ the ionic Mach number, with $v_{0}=\sqrt{\frac{k_{\mathrm{B}} T_{0}}{m_{\mathrm{i}}}}$ the ionic speed of sound,

$\xi=\frac{\vartheta_{0}}{v_{0}}$ the ratio of the typical velocity to the ionic speed of sound,

$\alpha=\frac{\vartheta_{0}}{c}$ the ratio of the typical velocity to the speed of light,

$\eta=\frac{q x_{0} E_{0}}{k_{\mathrm{B}} T_{0}}$ the ratio of the electric and plasma internal energies,

$\beta=\frac{\vartheta_{0} B_{0}}{E_{0}}$ the induced electric field relative to the total electric field,

$\kappa^{-1}=v_{\mathrm{ii}, 0} t_{0}$ the number of ion-ion collisions during the typical time. 
The dimensionless Maxwellians are defined by

$$
\begin{aligned}
\mathcal{M}_{n_{e}, u_{\alpha}, T_{\alpha}} & =\overline{\mathcal{M}}_{n_{e}, u_{\alpha}, T_{\alpha}} \\
& =n_{\mathrm{e}}(x, t)\left(\frac{1}{2 \pi T_{\alpha}(x, t)}\right)^{D_{v} / 2} \exp \left(-\frac{\left|M \varepsilon u_{\alpha}(x, t)-v\right|^{2}}{2 T_{\alpha}(x, t)}\right), \\
\mathcal{M}_{n_{i}, u_{\alpha}, T_{\alpha}} & =\overline{\mathcal{M}}_{n_{i}, u_{\alpha}, T_{\alpha}} \\
& =n_{\mathrm{i}}(x, t)\left(\frac{1}{2 \pi T_{\alpha}(x, t)}\right)^{D_{v} / 2} \exp \left(-\frac{\left|M u_{\alpha}(x, t)-v\right|^{2}}{2 T_{\alpha}(x, t)}\right) .
\end{aligned}
$$

Some comments can be stated regarding the meaning of these parameters and the scaling relations.

The typical mean velocity and temperature are assumed to be the same for electrons and ions. Accordingly, the relaxation of the electron mean velocity and temperature towards those of the ions may be assumed to marginally contribute to the evolution of the system. This assumption is therefore consistent with the investigation of resistive-less plasma modellings and the neglect of the inter-species collisions.

The parameter $\xi$ is intended to provide a measure of how the electronic and ionic dynamics are resolved. The choice $\xi=1$ means that the system is assumed to evolve at a speed comparable to the ionic thermal velocity $v_{0}$, while $\xi \varepsilon=1$ performs a rescaling of this typical velocity to the electron microscopic velocity. Setting $\xi=M$ relates the typical speed of the system to the ionic mean velocity $u_{0}$. Actually, the Mach number measures the gap between the microscopic (thermal) and macroscopic velocity scales.

The scaling relation $\eta=1$ is generally assumed in single-fluid plasma representation. The plasma internal energy is then on a par with the electric energy. This equilibrium is fundamental in the derivation of the Boltzmann relation. The identity $\beta M=\xi$ is also common in single-fluid plasma models. This amounts to assuming that the induced electric field scales as the product of the plasma mean velocity and the typical magnetic field: $E_{0}=$ $u_{0} B_{0}$. In other words, the magnetic field is essentially transported with the plasma flow. This latter assumption is in line with the Alfvén frozen theorem (Moreau 1990; Davidson 2001; Schnack 2009; Freidberg 2014) characteristic of ideal MHD models: the magnetic field is frozen into the plasma and transported by its flow.

The derivation of reduced models consists of identifying small dimensionless parameters and letting them go to zero. The smallness of the scaled Debye length refers to a typical space scale much larger than the physical Debye length. This means that the charge separations, occurring on space scales comparable to the Debye length, are assumed unimportant in explaining the evolution of the system. Sending the scaled Debye length to zero performs a low-frequency filtering into the equation deriving thus a quasi-neutral model. In the context of the derivation of numerical methods, the typical length relates to the mesh size. This outlines the advantage of reduced models: the low-frequency filtering operated by vanishingly small parameters permits the derivation of numerical methods with discretization parameters (mesh size and time step) unconstrained by the small scales filtered out from the original equations.

\section{A hierarchy of quasi-neutral models bridging the Vlasov-Maxwell system and the Hall MHD regime}

\subsection{Handling the fluid and quasi-neutral limits}

\subsubsection{A hierarchy of fluid and kinetic quasi-neutral models}

The aim here is to reduce the number of free dimensionless parameters, deriving by this means different reduced models well suited for the description of low-frequency 


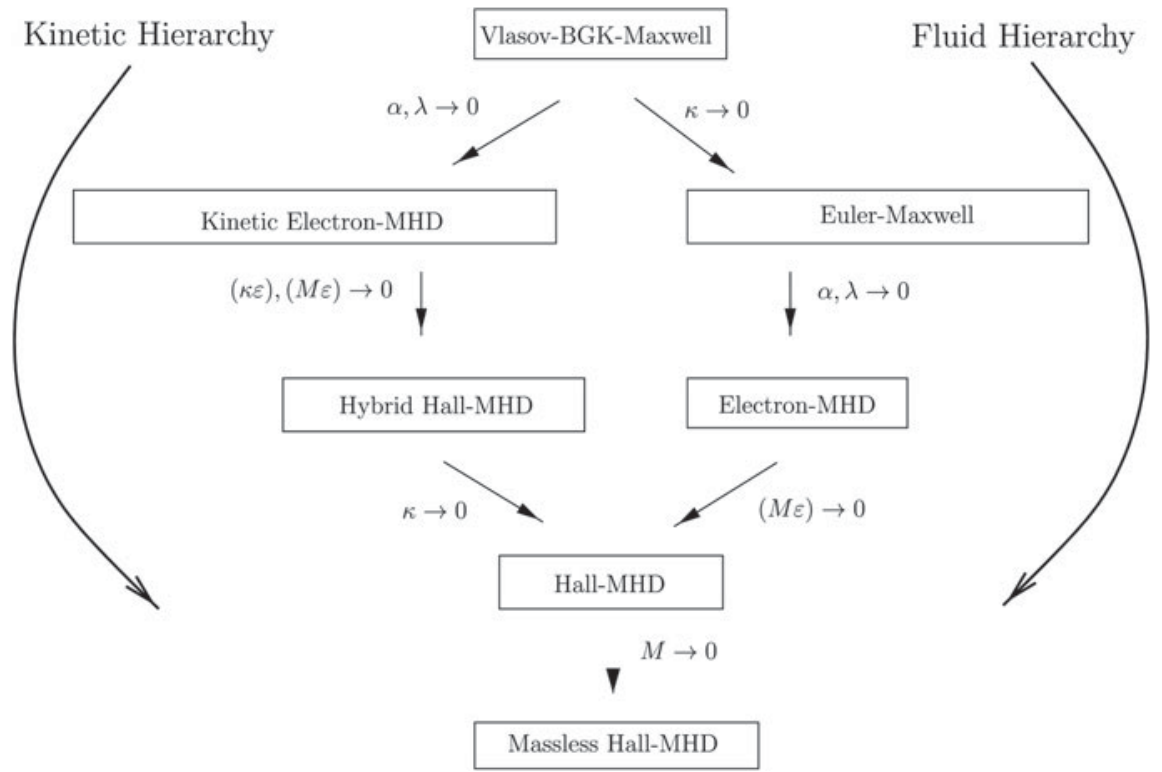

FIGURE 1. Fluid and kinetic (quasi-neutral) model hierarchies derived from the Vlasov-BGK-Maxwell system.

phenomena. As depicted in figure 1, the starting point of this hierarchy of models implements the minimal upgrades of the Vlasov-Maxwell system to recover a MHD regime. Precisely, only the inter-species collisions are taken into account in the initial model in order for the distribution function to relax towards the local equilibrium. This yields

$$
\begin{array}{r}
\xi \partial_{t} f_{\mathrm{i}}+v \cdot \nabla_{x} f_{\mathrm{i}}+\eta\left(E+\frac{\beta}{\xi} v \times B\right) \cdot \nabla_{v} f_{\mathrm{i}}=\frac{\xi}{\kappa} v_{\mathrm{ii}}\left(\mathcal{M}_{n_{\mathrm{i}}, u_{\mathrm{i}}, T_{\mathrm{i}}}-f_{\mathrm{i}}\right), \\
\xi \varepsilon \partial_{t} f_{\mathrm{e}}+v \cdot \nabla_{x} f_{\mathrm{e}}-\eta\left(E+\frac{\beta}{\varepsilon \xi} v \times B\right) \cdot \nabla_{v} f_{\mathrm{e}}=\frac{\xi}{\kappa} v_{\mathrm{ee}}\left(\mathcal{M}_{n_{\mathrm{e}}, u_{\mathrm{e}}, T_{\mathrm{e}}}-f_{\mathrm{e}}\right),
\end{array}
$$

for the evolution of the ions and electrons coupled to the dimensionless Maxwell system defined by (2.21).

From the scaling relations stated by $(2.18 a-c)$, discarding the inter-species collisions makes sense for the ions. Due to their large mass, the ions are almost unaffected by encounters with electrons. For the electrons, this assumption is not in line with the scaling of the intra- and inter-species collision frequencies. However, the purpose here is to propose a physically meaningful framework to clarify the foundation of a numerical method bridging the gap between a kinetic description of a weakly (or non-) collisional magnetized plasma with a MHD regime. The interspecies collisions give rise to the resistivity in the macroscopic system which is not the targeted class of modelling for this work.

\subsubsection{Handling the fluid limit}

To identify easily a fluid regime, the distribution function is decomposed into a Maxwellian $\mathcal{M}_{n_{\alpha}, u_{\alpha}, T_{\alpha}}$ and a deviation from this Maxwellian $\kappa g_{\alpha}$ according to

$$
f_{\alpha}=\mathcal{M}_{n_{\alpha}, u_{\alpha}, T_{\alpha}}+\kappa g_{\alpha},
$$


the deviation verifying

$$
\left\langle\left(\begin{array}{c}
1 \\
v \\
|v|^{2}
\end{array}\right) g_{\alpha}\right\rangle=\int_{\Omega_{v}}\left(\begin{array}{c}
1 \\
v \\
|v|^{2}
\end{array}\right) g_{\alpha} \mathrm{d} v=0 .
$$

With this decomposition, the Vlasov-Boltzmann equations (3.1) can be recast into a hydrodynamic set of equations with kinetic corrections, depending on the moment of the deviation $g_{\alpha}$, yielding

$$
\begin{gathered}
\frac{\xi}{M} \partial_{t} n_{\mathrm{i}}+\nabla_{x} \cdot\left(n_{\mathrm{i}} u_{\mathrm{i}}\right)=0, \\
M^{2}\left(\frac{\xi}{M} \partial_{t}\left(n_{\mathrm{i}} u_{\mathrm{i}}\right)+\nabla_{x} \cdot\left(n_{\mathrm{i}} u_{\mathrm{i}} \otimes u_{\mathrm{i}}\right)\right)+\nabla_{x} p_{\mathrm{i}}-\eta n_{\mathrm{i}}\left(E+\frac{\beta M}{\xi} u_{\mathrm{i}} \times B\right) \\
=-\kappa \nabla_{x} \cdot\left\langle v \otimes v g_{\mathrm{i}}\right\rangle, \\
\frac{\xi}{M} \partial_{t} W_{\mathrm{i}}+\nabla_{x} \cdot\left(\left(W_{\mathrm{i}}+p_{\mathrm{i}}\right) u_{\mathrm{i}}\right)-\eta n_{\mathrm{i}} E \cdot u_{\mathrm{i}}=-\frac{\kappa}{M} \nabla_{x} \cdot\left\langle\frac{|v|^{2}}{2} v g_{\mathrm{i}}\right\rangle,
\end{gathered}
$$

with

$$
W_{\mathrm{i}}=(M)^{2} \frac{1}{2} n_{\mathrm{i}}\left|u_{\mathrm{i}}\right|^{2}+\frac{p_{\mathrm{i}}}{\gamma-1}, \quad p_{\mathrm{i}}=n_{\mathrm{i}} T_{\mathrm{i}},
$$

for the ions, and an equivalent system for the electrons,

$$
\begin{gathered}
\frac{\xi}{M} \partial_{t} n_{\mathrm{e}}+\nabla_{x} \cdot\left(n_{\mathrm{e}} u_{\mathrm{e}}\right)=0, \\
(M \varepsilon)^{2}\left(\frac{\xi}{M} \partial_{t}\left(n_{\mathrm{e}} u_{\mathrm{e}}\right)+\nabla_{x} \cdot\left(n_{\mathrm{e}} u_{\mathrm{e}} \otimes u_{\mathrm{e}}\right)\right)+\nabla_{x} p_{\mathrm{e}}+\eta n_{\mathrm{e}}\left(E+\frac{\beta M}{\xi} u_{\mathrm{e}} \times B\right) \\
=-\kappa \nabla_{x} \cdot\left\langle v \otimes v g_{\mathrm{e}}\right\rangle, \\
\frac{\xi}{M} \partial_{t} W_{\mathrm{e}}+\nabla_{x} \cdot\left(\left(W_{\mathrm{e}}+p_{\mathrm{e}}\right) u_{\mathrm{e}}\right)+\eta n_{\mathrm{e}} E \cdot u_{\mathrm{e}}=-\frac{\kappa}{M \varepsilon} \nabla_{x} \cdot\left\langle\frac{|v|^{2}}{2} v g_{\mathrm{e}}\right\rangle,
\end{gathered}
$$

with

$$
W_{\mathrm{e}}=(M \varepsilon)^{2} \frac{1}{2} n_{\mathrm{e}}\left|u_{\mathrm{e}}\right|^{2}+\frac{p_{\mathrm{e}}}{\gamma-1}, \quad p_{\mathrm{e}}=n_{\mathrm{e}} T_{\mathrm{e}} .
$$

These two systems are coupled to a set of equations (the Maxwell system (2.21)) driving the changes in the electromagnetic field, as well as an equation for the evolution of the deviations $g_{\mathrm{e}}$ and $g_{\mathrm{i}}$. The construction of these equations will be omitted in the present work; we refer for instance to Crouseilles \& Lemou (2011), Crestetto et al. (2012) and De Cecco et al. (2017) for examples of their derivation.

\subsubsection{On the quasi-neutral limit}

Omitting the collisions, the fastest velocity in this system is the propagation of waves at the speed of light described by the Maxwell system. The Debye length as well as the plasma period also define small space and time scales for large plasma densities. 
The quasi-neutral limit is defined by the following scaling relations:

$$
(\alpha, \lambda) \rightarrow 0, \quad \alpha \sim \lambda .
$$

This amounts to assuming that the scaled Debye length is small compared to the typical length and that the system evolves at a speed lower than the speed of light. By this means, the small scales related to these parameters are filtered out of the equations. The last hypothesis $\alpha \sim \lambda$ is essential to recover the low-frequency Ampère law, derived by neglecting the displacement current. This equation being common to MHD models, the quasi-neutral limit encompasses these two assumptions. With the vanishing of this generalized dimensionless Debye length $(\lambda, \alpha) \rightarrow 0$, the Maxwell system degenerates into

$$
\begin{gathered}
\beta \nabla_{x} \times B=\frac{M}{\xi} J, \\
\beta \partial_{t} B+\nabla_{x} \times E=0, \\
n_{\mathrm{i}}=n_{\mathrm{e}}, \\
\nabla_{x} \cdot B=0 .
\end{gathered}
$$

From Gauss's law, the property of the electronic density to match that of the ions is recovered, which genuinely enforces quasi-neutrality of the plasma. The electric field has no contribution in either of these degenerate Gauss and Ampère equations. The remaining occurrence of the electric field is limited to the Faraday equation (3.6b). Therefore, this set of equations is not well suited for the computation of the electric field. Indeed, the electrostatic component of the electric field can be arbitrarily chosen in (3.6): the electric field satisfying this system may be augmented by any gradient of a scalar potential (see also Degond \& Deluzet (2017) for further details).

In the quasi-neutral limit, the electric field is provided by the particle current $J$ rather than the displacement current ( $\partial E / \partial t$ originally present in Ampère's law). To close the system, the dependence of $J$ with respect to $E$ shall be explained to restore uniqueness of the electric field. This is related to the model describing the plasma.

\subsection{A hierarchy of kinetic models for quasi-neutral plasmas}

\subsubsection{A kinetic formulation of the electron $M H D$}

The aim here is to follow the microscopic dynamics of the electrons. The velocity of interest is the kinetic velocity of the electrons. This amounts to setting $\vartheta_{0}=v_{0} / \varepsilon$ or equivalently $\xi \varepsilon=1$, yielding

$$
\begin{aligned}
\partial_{t} f_{\mathrm{i}}+\varepsilon\left(v \cdot \nabla_{x} f_{\mathrm{i}}+\eta(E+\varepsilon \beta v \times B) \cdot \nabla_{v} f_{\mathrm{i}}\right) & =\frac{1}{\kappa} \nu_{\mathrm{ii}}\left(\mathcal{M}_{n_{\mathrm{i}}, u_{\mathrm{i}}, T_{\mathrm{i}}}-f_{\mathrm{i}}\right), \\
\partial_{t} f_{\mathrm{e}}+v \cdot \nabla_{x} f_{\mathrm{e}}-\eta(E+\beta v \times B) \cdot \nabla_{v} f_{\mathrm{e}} & =\frac{1}{\varepsilon \kappa} \nu_{\mathrm{ee}}\left(\mathcal{M}_{n_{\mathrm{e}}, u_{\mathrm{e}}, T_{\mathrm{e}}}-f_{\mathrm{e}}\right) .
\end{aligned}
$$

The collisions are assumed to be ineffective on the characteristic time scale $\kappa \varepsilon \gg 1$, which amounts to neglecting the collision operator in (3.7), in particular for the ions, owing to $\varepsilon \ll 1$.

Performing the quasi-neutral limit $(\lambda=\alpha) \rightarrow 0$, the system at hand here is recast into (see Degond et al. 2015)

$$
\partial_{t} f_{\mathrm{i}}+\varepsilon\left(v \cdot \nabla_{x} f_{\mathrm{i}}+\eta(E+\varepsilon \beta v \times B) \cdot \nabla_{v} f_{\mathrm{i}}\right)=0,
$$




$$
\begin{gathered}
\partial_{t} f_{\mathrm{e}}+v \cdot \nabla_{x} f_{\mathrm{e}}-\eta(E+\beta v \times B) \cdot \nabla_{v} f_{\mathrm{e}}=0, \\
\beta \nabla_{x} \times B=(M \varepsilon) J, \\
\beta \partial_{t} B+\nabla_{x} \times E=0, \\
n_{\mathrm{e}}=n_{\mathrm{i}}=n, \\
\nabla_{x} \cdot B=0 .
\end{gathered}
$$

First, note that the formal time derivative of the Faraday equation (3.11) together with the curl of Ampère's law yields

$$
\nabla_{x} \times \nabla_{x} \times E=-(M \varepsilon) \partial_{t} J
$$

which outlines that the electric field is known up to the gradient of a potential in this system. In Degond, Deluzet \& Savelief (2012a) and Degond et al. (2015) the ill-posed nature of this equation is corrected by explaining the relation between the current density and the electric field. The conservation of the ionic and electronic momentum, as stated by $(3.3 b)$ and $(3.4 b)$, yields

$$
\begin{aligned}
(M \varepsilon) \frac{\partial J}{\partial t}= & -(M \varepsilon)^{2} \nabla_{x} \cdot\left(\mathbb{S}_{\mathrm{i}}-\mathbb{S}_{\mathrm{e}}\right)-\nabla_{x} \cdot\left(\varepsilon^{2} P_{\mathrm{i}}-P_{\mathrm{e}}\right) \\
& +\eta\left(\varepsilon^{2} n_{\mathrm{i}}+n_{\mathrm{e}}\right) E+\eta \beta(M \varepsilon)\left(\varepsilon^{2} n_{\mathrm{i}} u_{\mathrm{i}}+n_{\mathrm{e}} u_{\mathrm{e}}\right) \times B,
\end{aligned}
$$

with, for any species $\alpha$ ( $\alpha=\mathrm{e}$ for the electrons and $\mathrm{i}$ for the ions),

$$
\mathbb{S}_{\alpha}=n_{\alpha} u_{\alpha} \otimes u_{\alpha}, \quad P_{\alpha}=p_{\alpha} \amalg \mathrm{d}+\kappa\left\langle v \otimes v g_{\alpha}\right\rangle .
$$

Inserting the identity (3.15a), together with $n=n_{\mathrm{i}}=n_{\mathrm{e}}$, into (3.14) gives

$$
\begin{aligned}
n\left(1+\varepsilon^{2}\right) E+\nabla_{x} \times \nabla_{x} \times E= & -(M \varepsilon) n\left(\varepsilon^{2} u_{\mathrm{i}}+u_{\mathrm{e}}\right) \times B \\
& +(M \varepsilon)^{2} \nabla_{x} \cdot\left(\mathbb{S}_{\mathrm{i}}-\mathbb{S}_{\mathrm{e}}\right)+\nabla_{x} \cdot\left(\varepsilon^{2} P_{\mathrm{i}}-P_{\mathrm{e}}\right) .
\end{aligned}
$$

This equation is well posed in the quasi-neutral limit $(n>0)$ and can be used for the computation of the electric field. It is written under the assumption $\beta=1$ as well as $\eta=1$ which amounts to considering the thermal energy on a par with the electric one. This yields the following definition of the quasi-neutral model:

$$
\begin{gathered}
\partial_{t} f_{\mathrm{i}}+\varepsilon\left(v \cdot \nabla_{x} f_{\mathrm{i}}+(E+\varepsilon v \times B) \cdot \nabla_{v} f_{\mathrm{i}}\right)=0, \\
\partial_{t} f_{\mathrm{e}}+v \cdot \nabla_{x} f_{\mathrm{e}}-(E+v \times B) \cdot \nabla_{v} f_{\mathrm{e}}=0, \\
n\left(1+\varepsilon^{2}\right) E+\nabla_{x} \times \nabla_{x} \times E=-(M \varepsilon) n\left(\varepsilon^{2} u_{\mathrm{i}}+u_{\mathrm{e}}\right) \times B \\
+(M \varepsilon)^{2} \nabla_{x} \cdot\left(\mathbb{S}_{\mathrm{i}}-\mathbb{S}_{\mathrm{e}}\right)+\nabla_{x} \cdot\left(\varepsilon^{2} P_{\mathrm{i}}-P_{\mathrm{e}}\right), \\
\partial_{t} B+\nabla_{x} \times E=0, \\
\nabla_{x} \cdot B=0 .
\end{gathered}
$$

Note that the electric field provided by (3.17c) enforces a divergence-free particle current, or more precisely $\partial_{t}(\nabla \cdot J)=0$. This yields, because of the continuity equation,

$$
\frac{\partial^{2} \rho}{\partial t^{2}}=0
$$

This proves the consistency of this model with the quasi-neutrality assumption (matching of the electronic and ionic densities) as soon as the initial data are compliant with this 
regime. Note that, working a time semi-discretization, the vanishing of the charge density, rather than its time double derivative, may be recovered (see Degond et al. 2015). Note also that the evolution of the ions only brings a marginal correction, proportional to $\varepsilon^{2}$, to the electron dynamics. On this time scale, the ions may be considered at rest.

The characteristics of this model are similar to the so-called electron MHD: the time scale of interest is that of the electrons, the ions merely creating a motionless background for the fast electron flows (Kingsep, Chukbar \& Yank'kov 1990). In particular, this modelling accounts for the inertia of electrons. A noticeable difference from the electron MHD (see § 3.3.1) lies in the kinetic description of the plasma. An asymptotic-preserving method is proposed in Degond et al. (2015) to bridge this quasi-neutral model and the Vlasov-Maxwell system. The properties of this quasi-neutral plasma description are investigated in Tronci \& Camporeale (2015) by means of a linear stability analysis.

\subsubsection{A hybrid formulation of the Hall MHD}

Hybrid modelling (Yin et al. 2002; Winske et al. 2003; Tronci et al. 2014) refers to a class of plasma models where the ions are described by a kinetic equation while the fluid limit is assumed for the electrons. This is in line with the scaling relations of the collision frequencies stated by $(2.18 a-c)$. The relaxation of the electronic distribution function towards the local equilibrium is indeed faster than for the ions. The aim of this modelling is to filter out of the equations the fast scales carried by the electron dynamics. Therefore, a zero-inertia regime is also assumed for the electrons together with the fluid limit and the quasi-neutrality of the plasma.

The typical velocity selected here is the microscopic (thermal) velocity of the ions. This translates into the identity $\xi=1$ resulting in the following system for the plasma:

$$
\begin{gathered}
\partial_{t} f_{\mathrm{i}}+v \cdot \nabla_{x} f_{\mathrm{i}}+\eta\left(E+\frac{\beta}{\xi} v \times B\right) \cdot \nabla_{v} f_{\mathrm{i}}=\frac{1}{\kappa} v_{\mathrm{ii}}\left(\mathcal{M}_{n_{\mathrm{i}}, u_{\mathrm{i}}, T_{\mathrm{i}}}-f_{\mathrm{i}}\right), \\
\partial_{t} f_{\mathrm{e}}+\frac{1}{\varepsilon}\left(v \cdot \nabla_{x} f_{\mathrm{e}}-\eta\left(E+\frac{\beta}{\varepsilon \xi} v \times B\right) \cdot \nabla_{v} f_{\mathrm{e}}\right)=\frac{1}{\varepsilon \kappa} v_{\mathrm{ee}}\left(\mathcal{M}_{n_{\mathrm{e}}, u_{\mathrm{e}}, T_{\mathrm{e}}}-f_{\mathrm{e}}\right) .
\end{gathered}
$$

The fluid limit for the electrons is selected assuming $(\varepsilon \kappa) \ll 1$ meaning that the number of electron collisions during the typical time is large. The quasi-neutrality of the plasma amounts to setting $\lambda=\alpha \ll 1$. To overcome the degeneracy of the Maxwell system in the quasi-neutral limit, the electronic momentum is harnessed to provide the so-called generalized Ohm's law. The electronic system can be recast into

$$
\begin{aligned}
& (M \varepsilon)^{2}\left(\frac{1}{M} \partial_{t}\left(n_{\mathrm{e}} u_{\mathrm{e}}\right)+\nabla_{x} \cdot\left(n_{\mathrm{e}} u_{\mathrm{e}} \otimes u_{\mathrm{e}}\right)\right)+\nabla_{x} p_{\mathrm{e}}+\eta\left(n_{\mathrm{e}} E+(\beta M) u_{\mathrm{e}} \times B\right) \\
& \quad=-(\kappa(M \varepsilon)) \nabla_{x} \cdot \sigma_{\mathrm{e}} \\
& \frac{1}{M} \partial_{t} W_{\mathrm{e}}+\nabla_{x} \cdot\left(\left(W_{\mathrm{e}}+p_{\mathrm{e}}\right) u_{\mathrm{e}}\right)+\eta n_{\mathrm{e}} E \cdot u_{\mathrm{e}}=-\frac{\kappa}{M \varepsilon} \nabla_{x} \cdot\left((M \varepsilon)^{2} \sigma_{\mathrm{e}} \cdot u_{\mathrm{e}}+\mu_{\mathrm{e}} \nabla_{x} T_{\mathrm{e}}\right),
\end{aligned}
$$

where the viscous stress tensor $\sigma_{\mathrm{e}}$ as well as the thermal conductivity $\mu_{\mathrm{e}}$ are defined in $\S 3$ and, owing to the quasi-neutrality assumption, $n_{\mathrm{e}}=n_{\mathrm{i}}=n$.

The dynamics described by these equations is stiff. This is due to the smallness of $(\xi \varepsilon)$ in this regime: the thermal velocity of the ions (defined as the typical velocity) is small compared to that of the electrons. Therefore, the electrons are in a low-Mach regime. 
Assuming $(M \varepsilon) \ll 1$ gives rise to the following equilibria:

$$
\begin{gathered}
\nabla_{x}\left(n T_{\mathrm{e}}\right)+\eta n\left(E+(\beta M) u_{\mathrm{e}} \times B\right)=0, \\
\nabla_{x} T_{\mathrm{e}}=0 .
\end{gathered}
$$

The classical massless approximation for the electrons is recovered with the generalized Ohm's law and a homogeneous electronic temperature. The definition of the mean velocity $u_{\mathrm{e}}$ is derived from the particle current density $J=n\left(u_{\mathrm{i}}-u_{\mathrm{e}}\right)$ together with Ampère's law (3.10), yielding

$$
u_{\mathrm{e}}=u_{\mathrm{i}}-\frac{\beta}{M} \frac{\nabla_{x} \times B}{n} .
$$

The hybrid plasma modelling is written (assuming $\eta=\beta=1$ )

$$
\begin{gathered}
\partial_{t} f_{\mathrm{i}}+v \cdot \nabla_{x} f_{\mathrm{i}}+(E+v \times B) \cdot \nabla_{v} f_{\mathrm{i}}=0, \\
E=-M u \times B+M \frac{\nabla_{x} \times B}{n} \times B-T_{\mathrm{e}} \frac{\nabla_{x} n}{n}, \\
\frac{\partial B}{\partial t}+\nabla_{x} \times E=0, \quad \nabla_{x} \cdot B=0,
\end{gathered}
$$

with

$$
n=\int f_{\mathrm{i}} \mathrm{d} v, \quad n u=\int v f_{\mathrm{i}} \mathrm{d} v .
$$

The derivation of a similar model is proposed in Acheritogaray et al. (2011) with numerical investigations in Degond et al. (2011a).

\subsection{A fluid hierarchy of quasi-neutral models}

\subsubsection{The electron MHD system}

This model is obtained by letting $\kappa \rightarrow 0$ in (3.1). This yields the following set of equations for the electrons:

$$
\begin{gathered}
\frac{\xi}{M} \partial_{t} n_{\mathrm{e}}+\nabla_{x} \cdot\left(n_{\mathrm{e}} u_{\mathrm{e}}\right)=0, \\
\frac{\xi}{M} \partial_{t}\left(n_{\mathrm{e}} u_{\mathrm{e}}\right)+\nabla_{x} \cdot\left(n_{\mathrm{e}} u_{\mathrm{e}} \otimes u_{\mathrm{e}}\right)+\frac{1}{(M \varepsilon)^{2}}\left(\nabla_{x} p_{\mathrm{e}}+\eta n_{\mathrm{e}}\left(E+\frac{\beta M}{\xi} u_{\mathrm{e}} \times B\right)\right)=0 \\
\frac{\xi}{M} \partial_{t} W_{\mathrm{e}}+\nabla_{x} \cdot\left(\left(W_{\mathrm{e}}+p_{\mathrm{e}}\right) u_{\mathrm{e}}\right)+\eta n_{\mathrm{e}} E \cdot u_{\mathrm{e}}=0 .
\end{gathered}
$$

A similar system is derived for the ions, however with $\varepsilon=1$ and $\eta$ replaced by $-\eta$. These two sets of conservation laws are coupled to the Maxwell system (2.21). Performing the quasi-neutral limit in this system $(\lambda=\alpha) \rightarrow 0$ and focusing on the electronic dynamics with $\xi \varepsilon=1$ yields the quasi-neutral bi-fluid Euler-Maxwell system. This model is similar to that of $\$ 3.2 .1$ but with a fluid description for the plasma. Following the same terminology, this model is referred to as Electron-MHD system (also used in Kingsep et al. (1990) though different terminology may be used by other authors). It is implemented and investigated numerically in the framework of Asymptotic-Preserving methods in Degond et al. (2012a). 


\subsubsection{The Hall MHD regime}

The Hall MHD regime (see Lighthill 1960; Witalis 1986; Schnack 2009) is recovered from the assumptions of the previous section but with a typical velocity equal to the plasma mean flow yielding $\xi=M$. The fast electronic dynamics is filtered out from the equations to provide a low-frequency modelling for the plasma driven by the evolution of the massive ions. The plasma velocity, denoted $u$, is defined as that of the heavy species $u=u_{\mathrm{i}}$. The other parameters obey the classical scaling relations of MHD models: $\eta=1$ and $\beta=1$.

In the drift regime $(M \varepsilon \rightarrow 0)$, the electronic energy reduces to the internal energy

$$
\mathcal{E}_{\mathrm{e}}=p_{\mathrm{e}} /(\gamma-1)
$$

with the electronic momentum and energy verifying

$$
\begin{gathered}
\nabla_{x} p_{\mathrm{e}}=-n\left(E+u_{\mathrm{e}} \times B\right), \\
\partial_{t} \mathcal{E}_{\mathrm{e}}+\nabla_{x} \cdot\left(\left(\mathcal{E}_{\mathrm{e}}+p_{\mathrm{e}}\right) u_{\mathrm{e}}\right)=-n E \cdot u_{\mathrm{e}} .
\end{gathered}
$$

The generalized Ohm's law (3.26b) is harnessed to compute the electric field. The electronic velocity $u_{\mathrm{e}}$ is substituted by $u_{\mathrm{e}}=u-J / n$.

The electric field is computed via the generalized Ohm's law, giving rise to

$$
E=-u \times B+\frac{\nabla_{x} \times B}{n} \times B-\frac{\nabla_{x} p_{\mathrm{e}}}{n} .
$$

The first term on the right-hand side of equation (3.27) is the classical frozen field term, explaining the convection of the magnetic field together with the plasma. The second and third contributions are the so-called Hall and diamagnetic terms. Inserting this definition in the Faraday law (3.6b), the magnetic induction equation can be constructed, with

$$
\partial_{t} B+\nabla_{x} \cdot(u \otimes B-B \otimes u)=-\nabla_{x} \times\left(\frac{\nabla_{x} \times B}{n} \times B\right)+\nabla_{x} \times\left(\frac{\nabla_{x} p_{\mathrm{e}}}{n}\right) .
$$

Finally the plasma mass density, momentum and total pressure $p$ and energy $W$ defined by

$$
\begin{gathered}
p=p_{\mathrm{i}}+p_{\mathrm{e}}=n\left(T_{\mathrm{i}}+T_{\mathrm{e}}\right), \\
W=W_{\mathrm{i}}+\mathcal{E}_{\mathrm{e}}=(M)^{2} \frac{1}{2} n|u|^{2}+\frac{p}{\gamma-1}
\end{gathered}
$$

verify

$$
\begin{gathered}
\partial_{t} n+\nabla_{x} \cdot(n u)=0, \\
\partial_{t}(n u)+\nabla_{x} \cdot(n u \otimes u)+\frac{1}{M^{2}} \nabla_{x} p=\frac{1}{M^{2}} J \times B, \\
\partial_{t} W+\nabla_{x} \cdot((W+p) u)+\nabla_{x} \cdot\left(\left(\mathcal{E}_{\mathrm{e}}+p_{\mathrm{e}}\right) \mathrm{v}_{\mathrm{H}}\right)=E \cdot J .
\end{gathered}
$$

The Hall velocity $\mathrm{v}_{\mathrm{H}}$, which can be interpreted as the electron velocity in the ion frame, is defined by

$$
\mathrm{v}_{\mathrm{H}}=-\frac{J}{n} .
$$


The ideal MHD equations are classically written under a conservative form using the system total pressure and energy:

$$
p_{\text {ТОт }}=p+\frac{B^{2}}{2}, \quad W_{\text {ТОт }}=W+\frac{B^{2}}{2},
$$

writing the system as

$$
\begin{gathered}
\partial_{t} n+\nabla_{x} \cdot(n u)=0, \\
\partial_{t}(n u)+\nabla_{x} \cdot\left(n u \otimes u-\frac{1}{M^{2}} B \otimes B\right)+\frac{1}{M^{2}} \nabla_{x} p_{\mathrm{TOT}}=0, \\
\partial_{t} W_{\mathrm{TOT}}+\nabla_{x} \cdot\left(\left(W_{\mathrm{TOT}}+p_{\mathrm{TOT}}\right) u-(B \cdot u) B\right)=-\nabla_{x} \cdot\left(\left(\mathcal{E}_{\mathrm{e}}+p_{\mathrm{e}}\right) \mathrm{v}_{\mathrm{H}}\right), \\
\partial_{t} B-\nabla_{x} \cdot\left(B \otimes\left(u+\mathrm{v}_{\mathrm{H}}\right)-\left(u+\mathrm{v}_{\mathrm{H}}\right) \otimes B\right)=\nabla_{x} \times\left(\frac{\nabla_{x} p_{\mathrm{e}}}{n}\right) .
\end{gathered}
$$

This set of equations is supplemented with the electronic energy conservation $(3.26 c)$.

The ideal MHD equations are recovered from this system assuming an ideal Ohm's law where the current density is assumed small compared to the ion mean velocity and therefore neglected. However, in this simplified framework (omitting the unlike particle collisions), there are no mechanisms preventing the electron mean velocity from departing from that of the ions. Consequently, the generalized Ohm's law incorporates the Hall velocity in complement to the so-called ideal Ohm's law. The effect of the resistivity should be considered to derive the ideal MHD regime.

The drift approximation operated for the electrons amounts to vanishing of the electronic Mach numbers $(M \varepsilon)$. The scale separation introduced by the small electron to ion mass ratio $\varepsilon$ is not always sufficient to consider this limit independently of vanishing ionic Mach numbers $M \rightarrow 0$. For low ionic Mach number, a low-frequency filtering may be operated performing the limit of vanishing electronic Mach numbers jointly with ionic Mach numbers. This asymptotic defines the massless MHD regime (Besse et al. 2004).

\section{The electrostatic regime and the Boltzmann relation}

\subsection{Electrostatic limit of the Maxwell system}

The electrostatic regime is recovered from the dimensionless Maxwell system (2.21) by letting $\alpha$ go to zero, independently of $\lambda$. This assumption shall be interpreted as a typical velocity negligible compared to the speed of light. From Ampère's law

$$
\lambda^{2} \eta \frac{\partial E}{\partial t}+\frac{M}{\xi} J=\frac{\beta \lambda^{2} \eta}{\alpha^{2}} \nabla_{x} \times B,
$$

the limit $\alpha \rightarrow 0$ provides $\nabla \times B=0$. Nonetheless, the right-hand side of (4.1) remains an undetermined form. Therefore, Ampère's law is not well suited for the computation of the electric field in the electrostatic limit. However, subjected to convenient boundary conditions, the property $\nabla_{x} \times B=0$ together with $\nabla_{x} \cdot B=0$ yields $\partial_{t} B=0$. Inserting this property into the Faraday equation $(2.21 b)$ provides an electrostatic electric field: $\nabla \times E=0$. Furthermore, the undetermined form in (4.1) is divergence-free. Therefore, 
computing the divergence of Ampère's law provides

$$
\lambda^{2} \eta \frac{\partial \nabla_{x} \cdot E}{\partial t}=-\frac{M}{\xi} \nabla_{x} \cdot J,
$$

which is a well-posed problem for the electric field under the condition $\nabla_{x} \times E=0$. Note that, owing to the continuity equation

$$
\frac{\partial \rho}{\partial t}+\frac{M}{\xi} \nabla_{x} \cdot J=0,
$$

originating from the conservation of the particle densities (3.3a) and (3.4a), the divergence of Ampère's law is equivalent to the time derivative of Gauss's law, with

$$
\frac{\partial}{\partial t}\left(\lambda^{2} \eta \nabla_{x} \cdot E-\rho\right)=0 .
$$

Therefore, in the electrostatic regime, the Gauss equation is used to compute the electric field.

\subsection{Quasi-neutral models at the electronic scale}

This analysis is carried out under the assumption of a vanishing magnetic field $(B=0)$. The plasma description considered in the following is therefore

$$
\begin{gathered}
\xi \partial_{t} f_{\mathrm{i}}+v \cdot \nabla_{x} f_{\mathrm{i}}+\eta E \cdot \nabla_{v} f_{\mathrm{i}}=\frac{\xi}{\kappa}\left(\nu_{\mathrm{ii}}\left(\mathcal{M}_{n_{\mathrm{i}}, u_{\mathrm{i}}, T_{\mathrm{i}}}-f_{\mathrm{i}}\right)\right), \\
\xi \varepsilon \partial_{t} f_{\mathrm{e}}+v \cdot \nabla_{x} f_{\mathrm{e}}-\eta E \cdot \nabla_{v} f_{\mathrm{e}}=\frac{\xi}{\kappa}\left(\nu_{\mathrm{ee}}\left(\mathcal{M}_{n_{\mathrm{e}}, u_{\mathrm{e}}, T_{\mathrm{e}}}-f_{\mathrm{e}}\right)\right)
\end{gathered}
$$

coupled to the Gauss equation

$$
-\lambda^{2} \eta \Delta \phi=n_{\mathrm{i}}-n_{\mathrm{e}}
$$

$\phi$ being the electrostatic potential, with $E=-\nabla \phi$ and $n_{\alpha}=\int f_{\alpha} \mathrm{d} v$.

The quasi-neutrality of the plasma is recovered for vanishing scaled Debye length $\lambda \rightarrow 0$. In this regime, similarly to the electromagnetic case, an equation needs to be manufactured from the motion of the particles, to compute the electric field. This is classically obtained using the equation of the electronic momentum conservation. The electric field is computed in order for this conservation to be satisfied. In Degond et al. (2010) an equivalent approach is proposed. It consists of using the time derivatives of Gauss's law to produce

$$
-\lambda^{2} \eta \frac{\partial^{2}}{\partial t^{2}} \Delta \phi=\frac{\partial^{2}}{\partial t^{2}}\left(n_{\mathrm{i}}-n_{\mathrm{e}}\right) .
$$

In the quasi-neutral limit $(\lambda \rightarrow 0)$, the electrostatic field is the Lagrangian multiplier of the quasi-neutrality constraint

$$
\frac{\partial^{2}}{\partial t^{2}}\left(n_{\mathrm{i}}-n_{\mathrm{e}}\right)=0
$$

From the system (3.4) (with $B=0$ ), formally computing the time derivative of the density conservation together with the divergence of the momentum equation, the following 
identity is recovered:

$$
\begin{gathered}
\left(\frac{\xi}{M}\right)^{2} \frac{\partial^{2} n_{\mathrm{e}}}{\partial t^{2}}=-\nabla_{x} \cdot\left(\nabla_{x} \cdot\left(n_{\mathrm{e}} u_{\mathrm{e}} \otimes u_{\mathrm{e}}\right)\right)-\frac{1}{(M \varepsilon)^{2}} \nabla_{x} \cdot\left(\nabla_{x} P_{\mathrm{e}}+\eta n_{\mathrm{e}} E\right) \\
P_{\mathrm{e}}=p_{\mathrm{e}} \mathbb{I d}+\kappa\left\langle v \otimes v g_{\mathrm{e}}\right\rangle, \quad \text { with }\left\langle v \otimes v g_{\mathrm{e}}\right\rangle=\int(v \otimes v) g_{\mathrm{e}} \mathrm{d} v .
\end{gathered}
$$

Resuming the scaling relations of $\S 3.2 .1, \xi=1 / \varepsilon$ and $\kappa \varepsilon>1$, assuming that the ions are at rest, the evolution of the charge density $n_{\mathrm{i}}-n_{\mathrm{e}}$ is governed by (4.8) with

$$
\frac{\partial^{2} n_{\mathrm{e}}}{\partial t^{2}}=-(M \varepsilon)^{2} \nabla_{x} \cdot\left(\nabla_{x} \cdot\left(n_{\mathrm{e}} u_{\mathrm{e}} \otimes u_{\mathrm{e}}\right)\right)-\nabla_{x} \cdot\left(\nabla_{x} P_{\mathrm{e}}+\eta n_{\mathrm{e}} E\right) .
$$

The evolution of the density is barely independent of the mean flow velocity but relies on the balance between the pressure and the electric forces. The equation providing the electric potential $\phi$ is obtained by inserting this relation into (4.6) and passing to the limit $\lambda \rightarrow 0$.

This yields the following quasi-neutral kinetic plasma description:

$$
\begin{array}{r}
\partial_{t} f_{\mathrm{e}}+v \cdot \nabla_{x} f_{\mathrm{e}}+\eta \nabla_{x} \phi \cdot \nabla_{v} f_{\mathrm{e}}=\frac{1}{\varepsilon \kappa}\left(v_{e e}\left(\mathcal{M}_{n_{\mathrm{e}}, u_{\mathrm{e}}, T_{\mathrm{e}}}-f_{\mathrm{e}}\right),\right. \\
-\eta \nabla_{x} \cdot\left(n_{\mathrm{e}} \nabla_{x} \phi\right)=-\nabla_{x} \cdot\left(\nabla_{x} \cdot P_{\mathrm{e}}-(M \varepsilon)^{2} \nabla_{x} \cdot\left(n_{\mathrm{e}} u_{\mathrm{e}} \otimes u_{\mathrm{e}}\right)\right) .
\end{array}
$$

According to the values of $\kappa$, the collision term in (4.11a) may be disregarded, defining therefore a non-collisional kinetic description. Contrariwise, letting $\kappa \rightarrow 0$, a fluid description for the electrons may be derived, with

$$
\begin{gathered}
\frac{1}{(M \varepsilon)} \partial_{t} n_{\mathrm{e}}+\nabla_{x} \cdot\left(n_{\mathrm{e}} u_{\mathrm{e}}\right)=0, \\
\frac{1}{(M \varepsilon)} \partial_{t}\left(n_{\mathrm{e}} u_{\mathrm{e}}\right)+\nabla_{x} \cdot\left(n_{\mathrm{e}} u_{\mathrm{e}} \otimes u_{\mathrm{e}}\right)+\frac{1}{(M \varepsilon)^{2}}\left(\nabla_{x} p_{\mathrm{e}}-\eta n_{\mathrm{e}} \nabla_{x} \phi\right)=0, \\
\frac{1}{(M \varepsilon)} \partial_{t} W_{\mathrm{e}}+\nabla_{x} \cdot\left(\left(W_{\mathrm{e}}+p_{\mathrm{e}}\right) u_{\mathrm{e}}\right)-\eta n_{\mathrm{e}} \nabla_{x} \phi \cdot u_{\mathrm{e}}=0 .
\end{gathered}
$$

Note that a similar equation to (4.10), but with $P_{\mathrm{e}}=p_{\mathrm{e}} I \mathrm{~d}$, may be worked out of the conservation of the electronic density $(4.12 a)$ and momentum (4.12b). This outlines that the electronic dynamics, in particular the electronic speed of sound, is resolved in this model. Comparable models are implemented and numerically explored in the context of asymptotic-preserving methods in Crispel, Degond \& Vignal (2007) for fluid plasma description and Manfredi, Hirstoaga \& Devaux (2011) for kinetic equations.

\subsection{Quasi-neutral models at the ionic scale}

The typical velocity is chosen to be that of the ions with $\xi=1$ for the kinetic descriptions of the ions and $\xi=M$ for macroscopic models. 
The hybrid modelling investigated in $\S 3.2 .2$ is defined by the scaling relations $\xi=1$, $(\varepsilon \kappa) \ll 1$ and $\eta=1$. The equilibria stated by (3.22) yield

$$
T_{\mathrm{e}} \nabla_{x} n_{\mathrm{e}}=n_{\mathrm{e}} \nabla_{x} \phi
$$

with a homogeneous electronic temperature. This equation is integrated to provide the so-called Boltzmann relation

$$
n_{\mathrm{e}}=n_{0} \exp \left(-\frac{\phi}{T_{\mathrm{e}}}\right),
$$

$n_{0}$ being a constant (independent of the space variable $x$ ) that should be determined from adequate conditions (Hagelaar 2007). Due to the Boltzmann relation, the quasi-neutral limit is no longer singular. Indeed plugging the Boltzmann relation (4.14) into the Gauss equation yields

$$
-\lambda^{2} \Delta \phi=n_{\mathrm{i}}-n_{0} \exp \left(-\frac{\phi}{T_{\mathrm{e}}}\right) .
$$

This equation is not degenerate for the computation of the electric potential for vanishing $\lambda$. Indeed, the nonlinear part of the equation provides a means of computing $\phi$ in the quasi-neutral limit. This property is thoroughly investigated in Degond et al. (2012b).

The hybrid electrostatic model may be recast into

$$
\begin{gathered}
\partial_{t} f_{\mathrm{i}}+v \cdot \nabla_{x} f_{\mathrm{i}}-\nabla_{x} \phi \cdot \nabla_{v} f_{\mathrm{i}}=\frac{1}{\kappa}\left(v_{\mathrm{ii}}\left(\mathcal{M}_{n_{\mathrm{i}}, u_{\mathrm{i}}, T_{\mathrm{i}}}-f_{\mathrm{i}}\right)\right), \\
\phi=-T_{\mathrm{e}} \ln \left(\frac{n_{\mathrm{i}}}{n_{0}}\right) .
\end{gathered}
$$

Letting $\kappa \rightarrow 0$ together with $\kappa /(M \varepsilon) \ll 1$ and $\xi=M$ yields the quasi-neutral fluid model:

$$
\begin{gathered}
\partial_{t} n_{\mathrm{i}}+\nabla_{x} \cdot\left(n_{\mathrm{i}} u_{\mathrm{i}}\right)=0, \\
\partial_{t}\left(n_{\mathrm{i}} u_{\mathrm{i}}\right)+\nabla_{x} \cdot\left(n_{\mathrm{i}} u_{\mathrm{i}} \otimes u_{\mathrm{i}}\right)+\frac{1}{M^{2}}\left(\nabla_{x} p_{\mathrm{i}}+n_{\mathrm{i}} \nabla_{x} \phi\right)=0, \\
\partial_{t} W_{\mathrm{i}}+\nabla_{x} \cdot\left(\left(W_{\mathrm{i}}+p_{\mathrm{i}}\right) u_{\mathrm{i}}\right)+n_{\mathrm{i}} \nabla_{x} \phi \cdot u_{\mathrm{i}}=0, \\
\phi=-T_{\mathrm{e}} \ln \left(\frac{n_{\mathrm{i}}}{n_{0}}\right) .
\end{gathered}
$$

In the models (4.16) and (4.17) following the evolution of the plasma at the ionic scale, the fast electronic dynamics introduced by the electron inertia is filtered out of the equations by performing the low-frequency limit $(M \varepsilon) \rightarrow 0$.

\section{Conclusions}

In this paper, we propose an asymptotic analysis bridging kinetic plasma descriptions coupled to the Maxwell system and single-plasma modelling. Two frameworks are investigated. The first one is devoted to electromagnetic fields. The plasma is represented by a hierarchy of models starting with the bi-kinetic Vlasov-Maxwell system while ending with the single-fluid Hall MHD model. The second framework is dedicated to electrostatic fields. In this context, the asymptotic analysis permits the derivation of a hierarchy of models bridging the bi-kinetic Vlasov-Poisson system to a single-fluid representation 
consisting of a fluid system for the ions coupled to the Boltzmann relation for the electrons. The investigations proposed within this paper unravel different asymptotic parameters explaining the transition from one model to the other. The effort conducted in the present work consists of relating these asymptotic parameters to characteristics of the system. This means that the transition from one model to the other may be explained by a change in the plasma characteristics or the typical scales at which the plasma is observed.

This last notion is important in the perspective of designing a numerical method. Indeed, the discretization of these equations requires the use of a mesh interval as well as a time step. These two numerical parameters define the typical space and time scales, therefore a velocity scale as well, that the numerical method is aimed at capturing. This is related to the parameter $\xi$ used for the asymptotic analysis. Regarding the quasi-neutral modellings investigated, different choices are operated for this parameter. The fastest scales are related to the electron thermal velocity when the fast electron dynamics is intended to be captured by the model. This is for instance the value selected for electron MHD regimes, in either the fluid or kinetic frameworks. For hybrid or single-fluid plasma representations, the velocity scale is reduced to that of the mean flow of the plasma defined by the massive ions. The organization of $\S 4$ is aimed at emphasizing this feature.

The second parameter, already established in prior works (see Degond et al. 2010, $2012 a$, 2015), is the generalized scaled Debye length $\lambda$. It actually encompasses the scaled Debye length and the ratio of the typical velocity to the speed of light. Vanishing the generalized Debye length amounts to filtering out from the equations the small scales attached to the charge separation as well as those related to the propagation of electromagnetic waves at the speed of light. The quasi-neutral limit is therefore a low-frequency limit. Quasi-neutrality breakdowns may be explained by a refinement of the typical length scale or, for instance, a decrease of the plasma density. These changes are well accounted for by the asymptotic parameters set up to perform the analysis.

The vanishing of the electron inertia is related to a low-Mach regime $(M \varepsilon) \ll 1$. In single-fluid plasma representation, the fast electron dynamics is dropped out of the equations to perform a low-frequency filtering, the system being assumed to evolve at a lower speed attached to the massive ions. Nonetheless, the nature of the flow may be subjected to significant changes explaining that the particle inertia becomes significant again to account for the system evolution. This is illustrated in studies of plasma flows in sheaths, with supersonic particles, while the mean plasma velocity is small compared to the speed of sound in the plasma bulk (Chodura 1986; Stangeby 2000; Grismayer et al. 2008; Manfredi et al. 2008; Drouin et al. 2010). Accounting for this phenomenon is possible by selecting the appropriate typical velocity to resolve or filter the fast electron dynamics.

Finally the fluid assumption is classically related to a vanishing of the Knudsen number $\kappa \ll 1$ accounting for the relaxation of the distribution function towards the local thermodynamic equilibrium. The interplay of these four dimensionless parameters $(\xi, \lambda$, $M \varepsilon, \kappa)$ defines a hierarchy of reduced models bridging kinetic plasma descriptions coupled to the Maxwell system to quasi-neutral plasma representations including kinetic, hybrid and single-fluid modellings. Other reduced models may also be investigated considering different closures, to give access to a class of models with non-scalar pressure, or fluid models evolving higher-order moments. These low-frequency models are widely used to design efficient numerical methods. The benefit of the asymptotic analysis conducted within the study presented here draws the guidelines for the derivation of numerical methods implementing local up-scalings, therefore widening the use of numerical methods discretizing these reduced models. 


\section{Acknowledgements}

This work has been supported by Fédération de recherche pour la Fusion par Confinement Magnétique (FrFCM) in the frame of the project 'BRIDIPIC: BRIDging Particle-In-Cell methods and low frequency numerical models of plasmas'. A.C. acknowledges support from the French Agence Nationale pour la Recherche (ANR) in the frame of the projects MoHyCon ANR-17-CE40-0027-01 and MUFFIN ANR-19-CE46-0004.

Editor Giovanni Manfredi thanks the referees for their advice in evaluating this article.

Appendix A. Micro-macro decomposition, computation of the viscous terms

\section{A.1. Introduction, definitions, elementary properties}

The analyses carried out in this appendix are developed in the electrostatic framework and specified for electrons. The extensions for either electromagnetic fields or ions are straightforward and are therefore omitted for conciseness.

We first introduce the projector onto the Maxwellian. For any smooth function $\varphi$, the projector onto the Maxwellian denoted $\mathcal{M}_{\mathrm{e}}$ is

$$
\mathcal{M}_{\mathrm{e}}=n_{\mathrm{e}}(x, t) \frac{1}{\left(2 \pi T_{\mathrm{e}}(x, t)\right)^{D_{v} / 2}} \exp \left(\frac{\left|v-(M \varepsilon) u_{\mathrm{e}}(x, t)\right|^{2}}{2 T_{\mathrm{e}}(x, t)}\right) .
$$

For any smooth function $\varphi$, the projector onto $\mathcal{M}_{\mathrm{e}}$, denoted $\Pi_{\mathcal{M}_{\mathrm{e}}}$, is defined as (see Bennoune, Lemou \& Mieussens 2008; Crestetto et al. 2012)

$$
\begin{aligned}
\Pi_{\mathcal{M}_{\mathrm{e}}}(\varphi)= & {\left[\langle\varphi\rangle+\frac{\left(v-(M \varepsilon) u_{\mathrm{e}}\right)}{T_{\mathrm{e}}} \cdot\left\langle\left(v-(M \varepsilon) u_{\mathrm{e}}\right) \varphi\right\rangle\right.} \\
& \left.+\frac{2}{D_{v}}\left(\frac{\left|v-(M \varepsilon) u_{\mathrm{e}}\right|^{2}}{2 T_{\mathrm{e}}}-\frac{D_{v}}{2}\right)\left\langle\left(\frac{\left|v-(M \varepsilon) u_{\mathrm{e}}\right|^{2}}{2 T_{\mathrm{e}}}-\frac{D_{v}}{2}\right) \varphi\right\rangle\right] \frac{\mathcal{M}_{\mathrm{e}}}{n_{\mathrm{e}}},
\end{aligned}
$$

where $\langle\varphi\rangle=\int \varphi \mathrm{d} v$. For $k=1, \ldots, D_{v}$, we have the following properties:

$$
\begin{gathered}
\left(\mathbb{I d}-\Pi_{\mathcal{M}_{\mathrm{e}}}\right)\left(\partial_{t} \mathcal{M}_{\mathrm{e}}\right)=\left(\mathbb{I} \mathrm{d}-\Pi_{\mathcal{M}_{\mathrm{e}}}\right)\left(E \cdot \nabla_{v} \mathcal{M}_{\mathrm{e}}\right)=0 \\
\left(\mathbb{I} \mathrm{d}-\Pi_{\mathcal{M}_{\mathrm{e}}}\right)\left(\frac{1}{n_{\mathrm{e}}} \mathcal{M}_{\mathrm{e}} v_{k} \partial_{x_{k}} n_{\mathrm{e}}\right)=0 \\
\left(\mathbb{I d}-\Pi_{\mathcal{M}_{\mathrm{e}}}\right)\left(\left(v-(M \varepsilon) u_{\mathrm{e}}\right) \cdot \partial_{x_{k}} u_{\mathrm{e}} v_{k} \mathcal{M}_{\mathrm{e}}\right) \\
=\left(-\frac{\left|v-(M \varepsilon) u_{e}\right|^{2}}{D_{v}} \partial_{x_{k}} u_{e, k}+\left(v_{k}-(M \varepsilon) u_{e, k}\right)\left(v-(M \varepsilon) u_{k}\right) \cdot \partial_{x_{k}} u_{\mathrm{e}}\right) \mathcal{M}_{\mathrm{e}} \\
\left(\mathbb{I d}-\Pi_{\mathcal{M}_{\mathrm{e}}}\right)\left(\left(\frac{\left|v-(M \varepsilon) u_{\mathrm{e}}\right|^{2}}{2 T_{\mathrm{e}}^{2}}-\frac{D_{v}}{2 T_{\mathrm{e}}}\right) \mathcal{M}_{\mathrm{e}} v_{k} \partial_{x_{k}} T_{\mathrm{e}}\right) \\
=\mathcal{M}_{\mathrm{e}}\left(\frac{\left|v-(M \varepsilon) u_{\mathrm{e}}\right|^{2}}{2 T_{\mathrm{e}}^{2}}-\frac{D_{v}+2}{2 T_{\mathrm{e}}}\right)\left(v_{k}-(M \varepsilon) u_{\mathrm{e}, k}\right) \partial_{x_{k}} T_{\mathrm{e}} .
\end{gathered}
$$


Furthermore, if $g_{\mathrm{e}}$ satisfies the micro-macro decomposition (3.2), the following identity holds true:

$$
\Pi_{\mathcal{M}_{\mathrm{e}}}\left(g_{\mathrm{e}}\right)=\Pi_{\mathcal{M}_{\mathrm{e}}}\left(\partial_{t} g_{\mathrm{e}}\right)=0
$$

\section{A.2. Computation of the deviation to the Maxwellian}

The aim here is to characterize $g_{\mathrm{e}}$ or more specifically an approximation to the first order in $\kappa$. Inserting the micro-macro decomposition into the Vlasov-Boltzmann equation (4.5b) yields

$$
\xi \varepsilon \partial_{t} \mathcal{M}_{\mathrm{e}}+v \cdot \nabla_{x} \mathcal{M}_{\mathrm{e}}-\eta E \cdot \nabla_{v} \mathcal{M}_{\mathrm{e}}+\kappa\left(\xi \varepsilon \partial_{t} g_{\mathrm{e}}+v \cdot \nabla_{x} g_{\mathrm{e}}-\eta E \cdot \nabla_{v} g_{\mathrm{e}}\right)=\mathcal{L}_{M_{\mathrm{e}}} g_{\mathrm{e}}
$$

where

$$
\mathcal{L}_{M_{\mathrm{e}}} g_{\mathrm{e}}=-\xi v_{\mathrm{ee}} g_{\mathrm{e}}
$$

This provides, using properties (A 2),

$$
\mathcal{L}_{M_{\mathrm{e}}} g_{\mathrm{e}}=\left(\mathbb{I d}-\Pi_{\mathcal{M}_{\mathrm{e}}}\right)\left(v \cdot \nabla_{x} \mathcal{M}_{\mathrm{e}}\right)+\kappa\left(\xi \varepsilon \partial_{t} g_{\mathrm{e}}+\left(\mathbb{I d}-\Pi_{\mathcal{M}_{\mathrm{e}}}\right)\left(v \cdot \nabla_{x} g_{\mathrm{e}}-\eta E \cdot \nabla_{v} g_{\mathrm{e}}\right)\right) .
$$

It follows that

$$
g_{\mathrm{e}}=\left(\mathcal{L}_{M_{\mathrm{e}}}\right)^{-1}\left(\left(\mathbb{I d}-\Pi_{\mathcal{M}_{\mathrm{e}}}\right)\left(v \cdot \nabla_{x} \mathcal{M}_{\mathrm{e}}\right)+O(\kappa)\right)
$$

with

$$
v_{k} \partial_{x_{k}} \mathcal{M}_{\mathrm{e}}=\left(\frac{\partial_{x_{k}} n_{\mathrm{e}}}{n_{\mathrm{e}}}+\frac{M \varepsilon}{T_{\mathrm{e}}}\left(v-(M \varepsilon) u_{\mathrm{e}}\right) \cdot \partial_{x_{k}} u_{\mathrm{e}}+\left(\frac{\left(v-(M \varepsilon) u_{\mathrm{e}}\right)^{2}}{2 T_{\mathrm{e}}^{2}}-\frac{D_{v}}{2 T_{\mathrm{e}}}\right) \partial_{x_{k}} T_{\mathrm{e}}\right) v_{k} \mathcal{M}_{\mathrm{e}}
$$

From properties (A 2), we can state the expression of the deviation to the Maxwellian:

$$
\begin{aligned}
g_{\mathrm{e}}= & -\frac{\mathcal{M}_{\mathrm{e}}}{\xi v_{\mathrm{ee}}} \sum_{k=1}^{D_{v}}\left(\frac{M \varepsilon}{T_{\mathrm{e}}}\left(-\frac{\left|v-(M \varepsilon) u_{\mathrm{e}}\right|^{2}}{D_{v}} \partial_{x_{k}} u_{\mathrm{e}, k}+\left(v_{k}-(M \varepsilon) u_{\mathrm{e}, k}\right)\left(v-(M \varepsilon) u_{\mathrm{e}}\right) \cdot \partial_{x_{k}} u_{\mathrm{e}}\right)\right. \\
& \left.+\left(\frac{\left|v-(M \varepsilon) u_{\mathrm{e}}\right|^{2}}{2 T_{\mathrm{e}}^{2}}-\frac{D_{v}+2}{2 T_{\mathrm{e}}}\right)\left(v_{k}-(M \varepsilon) u_{\mathrm{e}, k}\right) \partial_{x_{k}} T_{\mathrm{e}}\right)+O\left(\frac{\kappa}{\xi}\right) .
\end{aligned}
$$

\section{A.3. Computation of the viscous terms}

The viscous terms are defined by

$$
\begin{gathered}
\left\langle v \otimes v g_{\mathrm{e}}\right\rangle=-\frac{M \varepsilon}{\xi} \sigma_{\mathrm{e}}+O\left(\frac{\kappa}{\xi}\right) \\
\left\langle\frac{|v|^{2}}{2} v g_{\mathrm{e}}\right\rangle=-\frac{1}{\xi}\left((M \varepsilon)^{2} \sigma_{\mathrm{e}} \cdot u_{\mathrm{e}}+q_{\mathrm{e}}\right)+O\left(\frac{\kappa}{\xi}\right) .
\end{gathered}
$$


Following the characterization (A 7) of $g_{\mathrm{e}}$, we can write

$$
\begin{aligned}
\sigma_{\mathrm{e}}= & -\frac{1}{T_{\mathrm{e}}} \frac{1}{v_{\mathrm{ee}}}\left(\left\langle-\frac{\left|v-(M \varepsilon) u_{\mathrm{e}}\right|^{2}}{D_{v}} \mathcal{M}_{\mathrm{e}}(v \otimes v)\right\rangle \sum_{l=1}^{D_{v}} \partial_{x_{l}} u_{\mathrm{e}, l}\right. \\
& \left.+\sum_{l=1}^{D_{v}}\left\langle(v \otimes v) \mathcal{M}_{\mathrm{e}}\left(v_{l}-(M \varepsilon) u_{\mathrm{e}, l}\right) \sum_{k=1}^{D_{v}}\left(v_{k}-(M \varepsilon) u_{\mathrm{e}, k}\right) \partial_{x_{l}} u_{\mathrm{e}, k}\right\rangle\right), \\
q_{\mathrm{e}}= & -\frac{1}{v_{\mathrm{ee}}}\left\langle\frac{|v|^{2}}{2} v \mathcal{M}_{\mathrm{e}}\left(\frac{\left|v-(M \varepsilon) u_{\mathrm{e}}\right|^{2}}{2 T_{\mathrm{e}}^{2}}-\frac{D_{v}+2}{2 T_{\mathrm{e}}}\right)\left(v-(M \varepsilon) u_{\mathrm{e}}\right) \cdot \nabla_{x} T_{\mathrm{e}}\right\rangle .
\end{aligned}
$$

Inserting in these definitions the following identities:

$$
\begin{aligned}
& \left\langle\frac{|v|^{2}}{2}(v \otimes v) \mathcal{M}_{\mathrm{e}}\left(\frac{\left|v-(M \varepsilon) u_{\mathrm{e}}\right|^{2}}{2 T_{\mathrm{e}}^{2}}+\frac{D_{v}+2}{2 T_{\mathrm{e}}}\right)\left(v-(M \varepsilon) u_{\mathrm{e}}\right) \cdot \nabla_{x} T_{\mathrm{e}}\right\rangle=0, \quad \text { (A 12) } \\
& -\frac{1}{T_{\mathrm{e}}} \frac{1}{v_{\mathrm{ee}}}\left(\left\langle\frac{\left|v-(M \varepsilon) u_{\mathrm{e}}\right|^{2}}{D_{v}}(v \otimes v) \mathcal{M}_{\mathrm{e}}\right\rangle \sum_{l=1}^{D_{v}} \partial_{x_{l}} u_{\mathrm{e}, l}\right. \\
& \left.-\sum_{l=1}^{D_{v}}\left\langle(v \otimes v) \mathcal{M}_{\mathrm{e}}\left(v_{l}-(M \varepsilon) u_{\mathrm{e}, l}\right) \sum_{k=1}^{D_{v}}\left(v_{k}-(M \varepsilon) u_{\mathrm{e}, k}\right) \partial_{x_{l}} u_{\mathrm{e}, k}\right\rangle\right)=(M \varepsilon) \sigma_{\mathrm{e}} \cdot u_{\mathrm{e}}
\end{aligned}
$$

we obtain

$$
\begin{gathered}
\sigma_{\mathrm{e}}=-\frac{1}{v_{\mathrm{ee}}}\left(n_{\mathrm{e}} T_{\mathrm{e}}\right)\left(\nabla_{x} u_{\mathrm{e}}+\nabla_{x} u_{\mathrm{e}}^{\mathrm{T}}-\frac{2}{D_{v}}\left(\nabla_{x} \cdot u_{\mathrm{e}}\right) \mathbb{I d}\right), \\
q_{\mathrm{e}}=-\frac{D_{v}+2}{2 v_{\mathrm{ee}}}\left(n_{\mathrm{e}} T_{\mathrm{e}}\right) \nabla_{x} T_{\mathrm{e}} .
\end{gathered}
$$

\section{REFERENCES}

Acheritogaray, M., Degond, P., Frouvelle, A. \& LiU, J.-G. 2011 Kinetic formulation and global existence for the hall-magneto-hydrodynamics system. Kinet. Relat. Models 4 (4), 901-918.

Bennoune, M., Lemou, M. \& Mieussens, L. 2008 Uniformly stable numerical schemes for the Boltzmann equation preserving the compressible Navier-Stokes asymptotics. J. Comput. Phys. 227 (8), 3781-3803.

Besse, C., Degond, P., Deluzet, F., Claudel, J., Gallice, G. \& Tessieras, C. 2004 A model hierarchy for ionospheric plasma modeling. Math. Models Meth. Appl. Sci. 14 (3), 393-415.

CHEN, G. \& CHACóN, L. 2015 A multi-dimensional, energy- and charge-conserving, nonlinearly implicit, electromagnetic Vlasov-Darwin particle-in-cell algorithm. Comput. Phys. Commun. 197, 73-87.

Chen, G., ChACóN, L. \& BARnes, D. C. 2011 An energy- and charge-conserving, implicit, electrostatic particle-in-cell algorithm. J. Comput. Phys. 230 (18), 7018-7036.

Chen, G., Chacón, L., Leibs, C. A., Knoll, D. A. \& Taitano, W. 2014 Fluid preconditioning for Newton-Krylov-based, fully implicit, electrostatic particle-in-cell simulations. J. Comput. Phys. 258, 555-567.

CHO, J. \& LAZARIAN, A. 2004 The anisotropy of electron MHD turbulence. Astrophys. J. 615, L41.

Chodura, R. 1986 Plasma flow in the sheath and the Presheath of a scrape-off layer. In Physics of Plasma-Wall Interactions in Controlled Fusion (ed. D. E. Post \& R. Behrisch), pp. 99-134. Springer. 
Crestetto, A., Crouseilles, N. \& Lemou, M. 2012 Kinetic/fluid micro-macro numerical schemes for Vlasov-Poisson-BGK equation using particles. Kinet. Relat. Models 5 (4), 787-816.

Crispel, P., Degond, P. \& Vignal, M.-H. 2007 An asymptotic preserving scheme for the two-fluid Euler-Poisson model in the quasineutral limit. J. Comput. Phys. 223 (1), 208-234.

Crouseilles, N. \& Lemou, M. 2011 An asymptotic preserving scheme based on a micro-macro decomposition for Collisional Vlasov equations: diffusion and high-field scaling limits. Kinet. Relat. Models 4 (2), 441-477.

DaldorfF, L. K. S., Töth, G., Gombosi, T. I., Lapenta, G., Amaya, J., Markidis, S. \& BRACKBILL, J. U. 2014 Two-way coupling of a global Hall magnetohydrodynamics model with a local implicit particle-in-cell model. J. Comput. Phys. 268, 236-254.

DAVIDson, P. A. 2001 An Introduction to Magnetohydrodynamics. Cambridge University Press.

De Cecco, A., Deluzet, F., Negulescu, C., Possanner, S. 2017 Asymptotic transition from kinetic to adiabatic electrons along magnetic field lines. Multiscale Model. Simul. 15 (1), 309-338.

DEGOND, P. 2007 Asymptotic continuum models for plasmas and disparate mass gaseous binary mixtures. In Material Substructures in Complex Bodies (ed. G. Capriz \& P. M. Mariano), chap. 1, pp. 1-62. Elsevier Science.

Degond, P. \& Deluzet, F. 2017 Asymptotic-preserving methods and multiscale models for plasma physics. J. Comput. Phys. 336, 429-457.

Degond, P., Deluzet, F., Dimarco, G., Gallice, G., Santagati, P. \& Tessieras, C. $2011 a$ Simulation of non equilibrium plasmas with a numerical noise reduced particle in cell method. In AIP Conference Proceedings, 27th Rarefied GAZ Dynamics, vol. 1333, pp. 1112-1117.

Degond, P., Deluzet, F. \& Doyen, D. 2015 Asymptotic-preserving particle-in-cell methods for the Vlasov-Maxwell system in the quasi-neutral limit. arXiv:1509.04235.

Degond, P., Deluzet, F. \& Navoret, L. 2006 An asymptotically stable particle-in-cell (PIC) scheme for collisionless plasma simulations near quasineutrality. C. R. Acad. Sci. Paris I 343 (9), 613-618.

Degond, P., Deluzet, F., Navoret, L., Sun, A.-B. \& Vignal, M.-H. 2010 Asymptotic-preserving particle-in-cell method for the Vlasov-Poisson system near quasineutrality. J. Comput. Phys. 229 (16), 5630-5652.

Degond, P., Deluzet, F. \& Savelief, D. 2012a Numerical approximation of the Euler-Maxwell model in the quasineutral limit. J. Comput. Phys. 231 (4), 1917-1946.

Degond, P., Dimarco, G. \& Pareschi, L. $2011 b$ The moment-guided Monte Carlo method. Intl J. Numer. Meth. Fluids 67 (2), 189-213.

Degond, P., Liu, H., Savelief, D. \& Vignal, M.-H. $2012 b$ Numerical approximation of the Euler-Poisson-Boltzmann model in the quasineutral limit. J. Sci. Comput. 51 (1), 59-86.

Dimarco, G., Mieussens, L. \& Rispoli, V. 2014 An asymptotic preserving automatic domain decomposition method for the Vlasov-Poisson-BGK system with applications to plasmas. J. Comput. Phys. 274, 122-139.

Drouin, M., Gremillet, L., AdAM, J.-C. \& HÉron, A. 2010 Particle-in-cell modeling of relativistic laser-plasma interaction with the adjustable-damping, direct implicit method. J. Comput. Phys. 229 (12), 4781-4812.

FreidberG, J. P. 2014 Ideal MHD. Cambridge University Press.

Gordeev, A. V., Kingsep, A. S. \& Rudakov, L. I. 1994 Electron magnetohydrodynamics. Phys. Rep. 243 (5), 215-315.

Greene, J. M. 1973 Improved Bhatnagar-Gross-Krook model of electron-ion collisions. Phys. Fluids 16 (11), 2022.

Grismayer, T., Mora, P., AdAM, J. \& Héron, A. 2008 Electron kinetic effects in plasma expansion and ion acceleration. Phys. Rev. E 77 (6), 066407.

HagelaAR, G. J. M. 2007 How to normalize Maxwell-Boltzmann electrons in transient plasma models. J. Comput. Phys. 227 (2), 871-876.

HUBA, J. D. 2011 NRL plasma formulary. Tech. Rep. Naval Research Laboratory.

JANG, J. \& MASMOUdi, N. 2012 Derivation of Ohm's law from the kinetic equations. SIAM J. Math. Anal. 44 (5), 3649-3669. 
JIN, S. 1999 Efficient asymptotic-preserving (AP) schemes for some multiscale kinetic equations. SIAM J. Sci. Comput. 21 (2), 441-454.

Kingsep, A. S., Chukbar, K. V. \& YAnK'Kov, V. V. 1990 Electron magnetohydrodynamics. In Reviews of Plasma Physics, vol. 16, pp. 243-291. Springer.

Klingenberg, C., Pirner, M. \& Puppo, G. 2016 A consistent kinetic model for a two-component mixture with an application to plasma. Kinet. Relat. Models 10 (2), 445-465.

LANGMUIR, I. 1929 The interaction of electron and positive ion space charges in cathode sheaths. Phys. Rev. 33 (6), 954-989.

Lemou, M. \& Mieussens, L. 2008 A new asymptotic preserving scheme based on micro-macro formulation for linear kinetic equations in the diffusion limit. SIAM J. Sci. Comput. 31 (1), 334-368.

LighthiLl, M. J. 1960 Studies on magneto-hydrodynamic waves and other anisotropic wave motions. Phil. Trans. R. Soc. Lond. A 252 (1014), 397-430.

Manfredi, G., Devaux, S., Shukla, P. K., Eliasson, B. \& Stenflo, L. 2008 Plasma-wall transition in weakly collisional plasmas. In AIP Conference Proceedings, pp. 178-187. AIP.

MAnfredi, G., HirstoagA, S. \& DevauX, S. 2011 Vlasov modelling of parallel transport in a tokamak scrape-off layer. Plasma Phys. Control. Fusion 53 (1), 015012.

Moreau, R. J. 1990 Magnetohydrodynamics, 1990th edn. Springer.

Park, W., Parker, S., Biglari, H., Chance, M., Chen, L., Cheng, C. Z., Hahm, T. S., Lee, W. W., Kulsrud, R., Monticello, D. et al. 1992 Three-dimensional hybrid gyrokinetic-magnetohydrodynamics simulation. Phys. Fluids B 4 (7), 2033-2037.

SCHNACK, D. D. 2009Lectures in Magnetohydrodynamics, Lecture Notes in Physics, vol. 780. Springer.

Schumer, J., Swanekamp, S., Ottinger, P., Commisso, R., Weber, B., Smithe, D. N. \& LUDEKING, L. 2001 MHD-to-PIC transition for modeling of conduction and opening in a plasma opening switch. IEEE Trans. Plasma Sci. 29 (3), 479-493.

SpatscheK, K.-H. 2012 High Temperature Plasmas: Theory and Mathematical Tools for Laser and Fusion Plasmas. Wiley-VCH.

Stangeby, P. C. 2000 The Plasma Boundary of Magnetic Fusion Devices. Institute of Physics Pub.

Swanekamp, S. B., Grossmann, J. M., Fruchtman, A., Oliver, B. V. \& Ottinger, P. F. 1996 Particle-in-cell simulations of fast magnetic field penetration into plasmas due to the hall electric field. Phys. Plasmas 3 (10), 3556-3563.

TONKS, L. \& LANGMUIR, I. 1929a A general theory of the plasma of an arc. Phys. Rev. 34 (6), 876-922.

TONKs, L. \& LANGMUIR, I. 1929 b Oscillations in ionized gases. Phys. Rev. 33 (2), 195-210.

Tronci, C. \& CAmporeale, E. 2015 Neutral Vlasov kinetic theory of magnetized plasmas. Phys. Plasmas 22 (2), 020704.

Tronci, C., Tassi, E., Camporeale, E. \& Morrison, P. J. 2014 Hybrid Vlasov-MHD models: Hamiltonian vs. non-Hamiltonian. Plasma Phys. Control. Fusion 56 (9), 095008.

WinsKe, D., YIn, L., OMIDI, N., KARIMABADI, H. \& QUEST, K. 2003Hybrid simulation codes: past, present and future-a tutorial. In Space Plasma Simulation (ed. J. Büchner, M. Scholer \& C. T. Dum), vol. 615, pp. 136-165. Springer.

WitALIS, E. 1986 Hall magnetohydrodynamics and its applications to laboratory and cosmic plasma. IEEE Trans. Plasma Sci. 14 (6), 842-848.

Yin, L., Winske, D., GARY, S. P. \& BIRN, J. 2002 Hybrid and hall-magnetohydrodynamics simulations of collisionless reconnection: effect of the ion pressure tensor and particle Hall-magnetohydrodynamics. Phys. Plasmas 9 (6), 2575. 